\title{
The triangular self in the social media era
}

\author{
Qi Wang (1) \\ Cornell University, Ithaca, NY, USA \\ Corresponding author: Qi Wang, email: qiwang@cornell.edu
}

(Received 16 September 2021; accepted 17 September 2021)

\begin{abstract}
I propose a triangular theory of self to characterise the sense of selfhood in the era of social media. According to the theory, the self in the social media era comprises the represented self that is located in the private mind of the person, the registered self that is presented on social media platforms, and the inferred self that is constructed by the virtual audience. The three components of the self interact in dynamic ways to constitute a sense of selfhood and identity specific to the social media era. Autobiographical memory plays a critical role in the development and maintenance of these components. The triangular theory of self introduces new ways to understand and study memory and self in a digitally mediated world.
\end{abstract}

Keywords: triangular theory of self; self; social media; Internet; autobiographical memory; transactive mind

Our sense of self evolves as our society changes. With the vast advances of digital technology over the past decades, the growing impact of social media has been exponential on how we see the world and how we view ourselves (Auxier and Anderson 2021; Dean 2021). Not only has social media become a major source for individuals to receive information, but it has also served as a democratised forum for individuals to create and disseminate information. Half of the 7.7 billion people around the globe are active users on social media platforms such as Facebook, Twitter, WhatsApp, and WeChat. Just Facebook alone, there are approximately 2 billion daily active users who produce 293,000 status updates and 510,000 comments every minute (Dean 2021; Marr 2021). Particularly important in the current context, social media affords individuals with new means to share their personal experiences with a wide range of audiences physically afar (Bell and Gemmell 2010; Lenhart and Fox 2006; Stone and Wang 2019). This contributes to a sense of selfhood that is unprecedented in modern times (Wang 2013, 2021). In this article, I propose a triangular theory of self to characterise the sense of selfhood unique in the era of social media.

\section{The triangular theory of self}

The self is a multifaceted, complex, and dynamic construct that encompasses an individual's subjective experience of the world and aggregated knowledge of one's own traits

(c) The Author(s), 2021. Published by Cambridge University Press. This is an Open Access article, distributed under the terms of the Creative Commons Attribution licence (http://creativecommons.org/licenses/by/4.0/), which permits unrestricted re-use, distribution and reproduction, provided the original article is properly cited. 


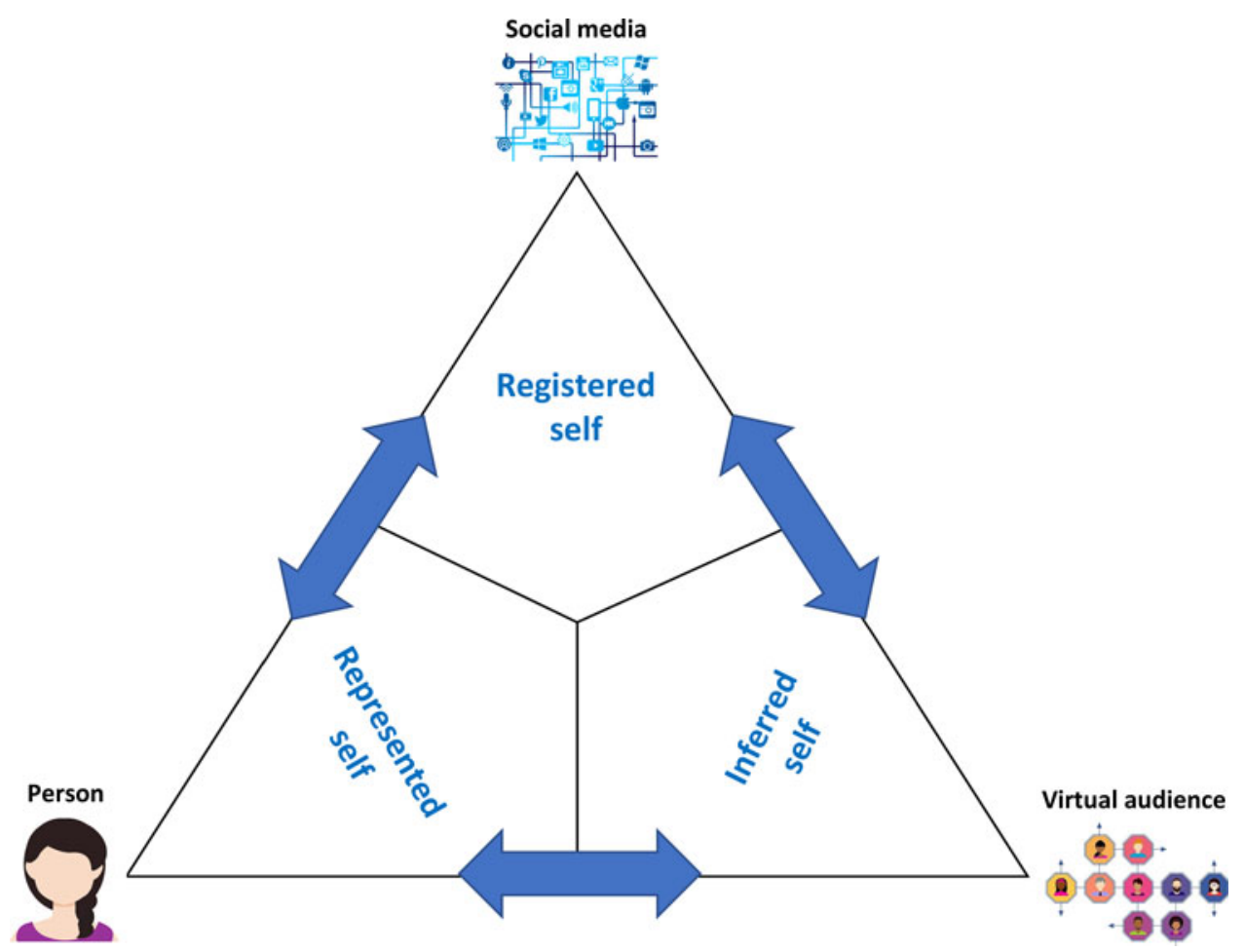

Fig. I. The triangular self in the social media era.

and attributes (James 1890; Neisser 1988; Truong and Todd 2017). Autobiographical memory, or memory for significant personal experiences from an individual's life, further supplements the self with a temporal dimension and contributes to a sense of selfcontinuity over time (Fivush 2011; Wang 2013). The self is characterised by reflective consciousness that is essentially private to the individual mind and supported by specific neural networks (Modinos et al 2011; Pfeifer et al 2007). In the meantime, the self operates in a social world and is marked by interpersonal roles and reputation; as such, the self is highly responsive to the views of others and to the sociocultural context (Wang 2006; Wang and Chaudhary 2006).

The dynamic characteristics of the self are accentuated in the digitally mediated modern society (Wang 2013). According to the triangular theory, the self in the social media era is composed of three types of representations: the represented self, the registered self, and the inferred self. The represented self is the characteristics, roles, and experiences of oneself as perceived and encoded by the person who acts as an agentic experiencer and knower. The registered self is the characteristics, roles, and experiences of the person as shared on social media. The inferred self is the characteristics, roles, and experiences of the person as viewed and interpreted by the virtual audience. The represented self, while in line with the traditional notion of selfhood (James 1890; Neisser 1988; Truong and Todd 2017), is externalised in the social media era to the registered self as its digital extension and to the inferred self as its transactive extension. The three components of the self mutually influence each other to constitute a sense of selfhood and identity that is specific to the social media era. The theoretical model is depicted in Figure 1. I discuss each component of the triangular self in turn, as well as the interplay between the three components and between the person, social media, and the virtual audience. 


\section{The represented self and its externalisation}

In the triangular theory of self, the represented self refers to one's subjective selfrepresentations - including representations of autobiographical experiences - that are 'located' in the private mind and brain of the person. Yet, the represented self is embedded and constructed in the sociocultural context and, in the social media era, it is externalised and outsourced to cyberspace, where the person selectively processes, encodes, and shares self-related information online (e.g., personal events, photos, opinions, ideas, etc.) (Wang 2013, 2019). The development and maintenance of the represented self, therefore, entails an active, dynamic process in which the person shares his or her experiences and views on selected social media platforms with an intended virtual audience who are often physically afar and psychologically heterogeneous. Different from face-to-face communication and memory sharing, sharing personal experiences online is not constrained by the physical location or immediate availability of listeners but can be achieved at any time with a large number of people with varying gradations in relationship closeness - family, friends, acquaintances, and strangers (Stone and Wang 2019; Wang 2018).

The public sphere, the unlimited audience, and the permanent storage of the shared information in cyberspace make the motives of online sharing activities simultaneously personal and social. For instance, bloggers, who are dubbed as the 'Internet's storytellers' (Lenhart and Fox 2006), use social media as a tool to both express themselves and connect with others. Sharing with the public what is traditionally considered private and diary-only information, many bloggers view blogging as a personal endeavour for themselves. In the meantime, they expect feedback from their audience, and some post material primarily to engage their intended audience such as friends and family (Lenhart and Fox 2006; Stefanone and Jang 2008).

General memory research has shown that people share memories for various purposes: to convey information about who they are - the self function, to entertain others and develop and maintain relationships - the social function, to seek empathy and support for emotion regulation and coping - the therapeutic function, and to impart lessons to help others - the directive function (e.g., Alea and Bluck 2003; Kulkofsky et al 2010; Pillemer 2001). In particular, social connection is the most prominent purpose of fact-to-face memory sharing (Alea and Bluck 2003; Guan and Wang 2020; Kulkofsky et al 2010). In the context of social media, people share memories for similar reasons (Hollenbaugh 2011; Wang 2013). For example, in response to the Purposes of Online Memory Sharing Scale (POMSS), adults of different ages report sharing personal experiences on social media most primarily for social reasons, followed by self-reasons and then directive and therapeutic reasons (see Figure 2; Stone et al 2021; Wang 2020). People who endorse more strongly the various purposes of online memory sharing post more frequently personal experiences on social media (Wang 2020). There are also individual differences. For instance, individuals with a greater disclosure tendency endorse more strongly all purposes of online memory sharing; individuals who are more interdependent report online sharing more for social and directive purposes; and those who experience greater loneliness report online sharing more for therapeutic reasons (Stone et al 2021).

Sharing personal experiences online, in turn, has consequences for the represented self. Unlike the online offloading of factual information which often impairs subsequent recall (Ferguson et al 2015; Kahn and Martinez 2020; Sparrow et al 2011), sharing personal information on social media may provide opportunities for rehearsal and meaning making and, in turn, facilitate long-term memory (Stone and Wang 2019). In two studies that investigated this question, participants completed a daily diary for a week. They recorded 


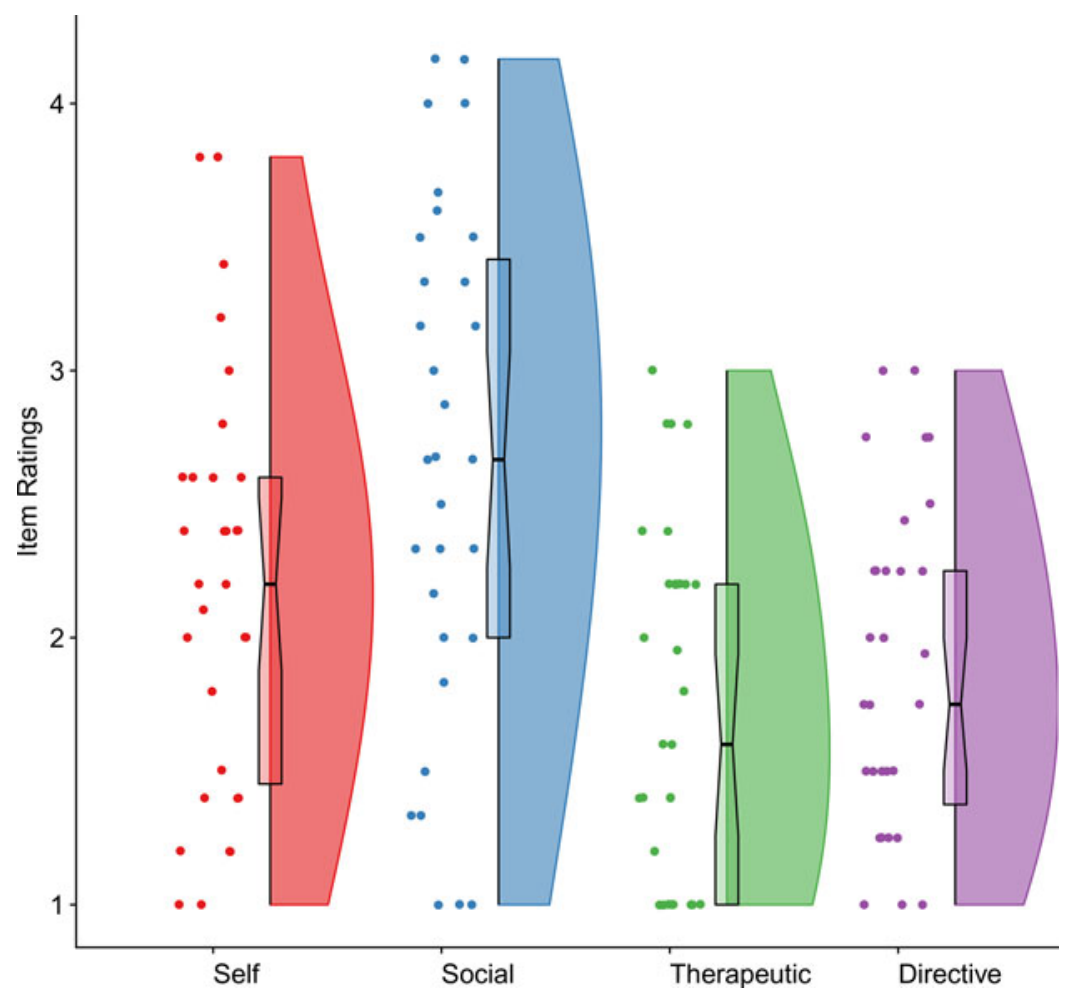

Fig. 2. Purposes of online memory sharing. The raincloud plots' display the data distribution, interquartile range, and median of the ratings on each purpose. Data from Wang (2020).

autobiographical events each day and were instructed to keep note of whether they shared any of the events online (Wang et al 2017) or to use or refrain from using Snapchat on alternate days (Johnson and Morley 2021). At the completion of the diary recording, participants were more likely to remember events that they had posted on social media than those not posted (Wang et al 2017), and they correctly recalled more events from the Snapchat days than the non-Snapchat days (Johnson and Morley 2021). Online sharing, thus, enhances recall of autobiographical events.

The influence of social media and the virtual audience on the represented self is complex and multifaceted (Wang 2013). Sharing memories online with a diverse audience facilitates interpersonal connection and personal expression (Hollenbaugh 2011; Wang 2013), while private reminiscing often focuses on reflecting on the past for future guidance (Kulkofsky et al 2010). Given the presence versus absence of the virtual audience and the different retrieval contexts and goals, event details posted online and those recalled privately may exhibit inconsistency. Over time, however, as the original memory traces start to fade, the wide variety of memory cues online such as comments, Likes, and periodic reminders may facilitate retention of the posted event details. As a result, event details shared in social media posts may become stabilised and remembered, while those not shared may be forgotten or inaccessible (Stone and Wang 2019).

In a laboratory experiment to test whether memory details shared online would persist in later offline recall, participants were asked to generate recent life events in response to cue words and then describe the event details as if they were writing about the events either on WeChat or in their diaries (2021). Participants received a surprise memory 
test for the events either one week or two weeks later, either with or without the original cue words. It was found that, following a one-week interval, participants who initially retrieved memories on WeChat recalled more inconsistent details and more commissions than those who initially retrieved memories in a private diary. However, the differences dissipated and even reversed among participants following a two-week interval and with the assistance of cues. It appears that although memories shared online are subject to reconstruction deviating from offline recall, as time goes by, feedbacks and reminders on social media platforms may contribute to a 'narrow version' of our autobiographical memories in alignment with the online posts.

Taken together, the represented self is receptive to the context of social media and the virtual community. Although private to the individual mind, it is externalised in the social media era, a motivated process that, in turn, shapes self-representations. The person plays an active role in creating the digital extension of the self that is registered online.

\section{The registered self and its infinite possibilities}

The registered self is the online presentations of one's autobiographical experiences and the associated personal characteristics and roles, enabled by social media platforms (Figure 1). Unlike the represented self, the registered self is public by default and interactive in nature. The registered self may bear some resemblance with what is traditionally portrayed in memoirs or autobiographies, whereby, in both cases, the person tells his or her stories to an intended audience (Wang 2018). Yet social media makes the registered self a democratised outlet of self-presentation for all, rather than a chosen few, encoded in infinite and permanent e-memory. Furthermore, the functional capabilities of social media platforms provide rich technology features for information sharing in multimedia forms, such as text, photo, video, hyperlink, and even livestreaming and augmented reality. This affords individuals with infinite possibilities to create a digital extension of the self. The public sphere and the technological capacity of social media give rise to distinct characteristics of the registered self, with its content and form serving the person's goals to communicate the self and relate to other selves online (Figure 3; for detailed discussion, see Wang 2013).

Specifically, the registered self is constructed with the virtual audience in mind and is, therefore, dialogical in nature. The person may use a variety of tactics to engage the audience, such as to provide captivating details of his or her experiences and to directly ask questions or seek feedback (Miura and Yamashita 2007). Also, sustained by a wide array of multimedia strategies, the registered self often portrays a unique persona distinctly different from other selves online. It is a singular selfhood with maximised individuality, who authors one's own life stories and acts as the central character (Serfaty 2004). Furthermore, the registered self is often expressive and even disclosive, revealing intimate personal information and innermost thoughts and feelings, a critical component in the development of interpersonal closeness and social bonding (Levinger 1980). Online selfdisclosure is especially common among extroverts and those who exhibit disclosiveness traits (Hollenbaugh 2011; Stefanone and Jang 2008). The registered self is also performed, being presented in a positive light to express an ideal self (Lee et al 2008). In line with these characteristics of the registered self, autobiographical memories recalled for online posting tend to be more elaborate, more likely to include questions, more self-revealing, and more positive, when compared with memories recalled for a private diary (Wang et al 2015).

The role of microblogging (e.g., status updates) as a social media genre in representing the registered self is particularly notable. During microblogging, individuals make short, frequent posts for online audience interactions in the format of text as well as links, audio, 


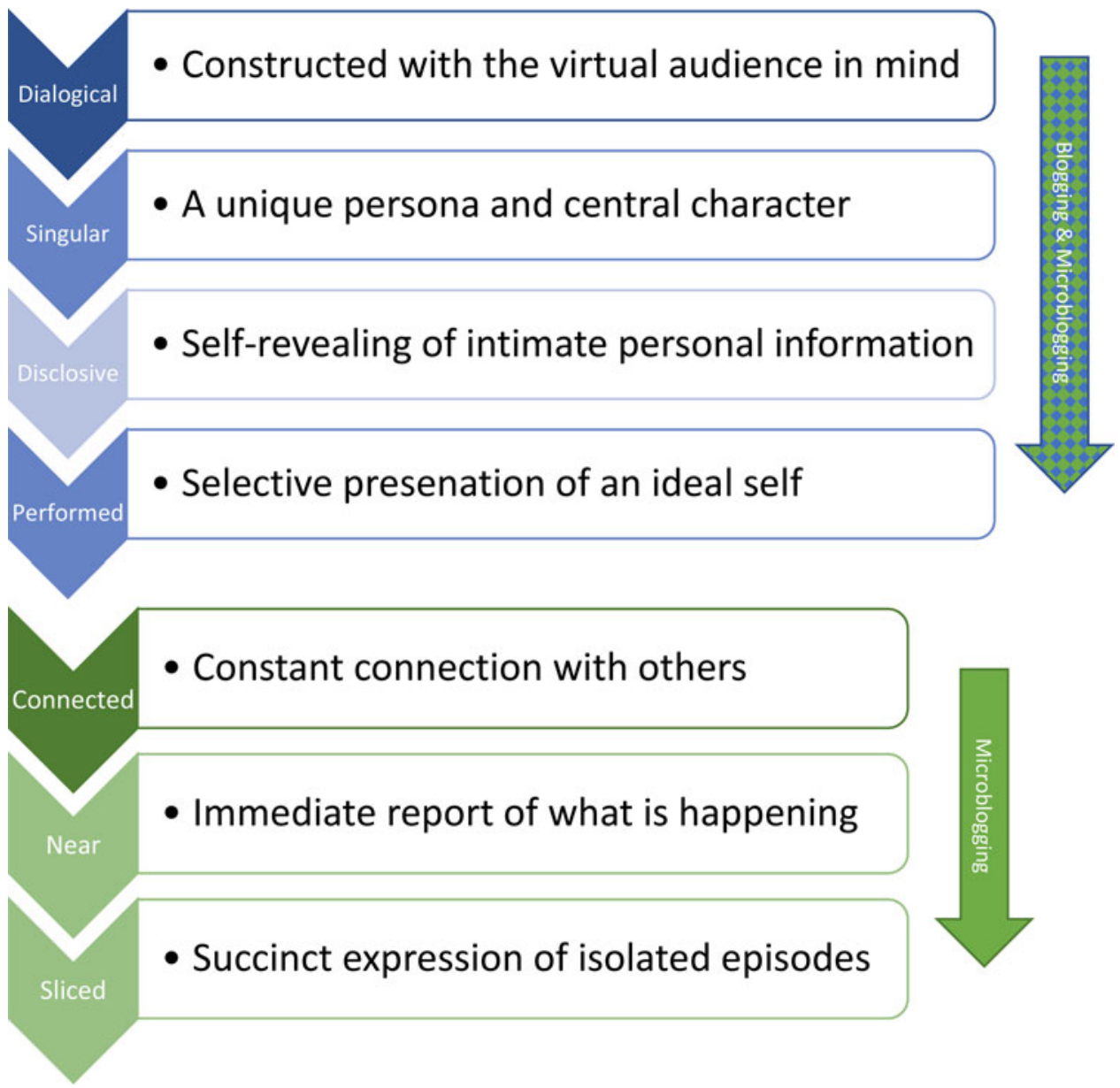

Fig. 3. Distinct characteristics of the registered self on social media.

images, and video. In addition to sharing information (e.g., what is in the news), individuals post their moment-to-moment whereabouts and trivial ephemera. Compared with traditional blogging, microblogging is a faster, more convenient, and more palatable way of communicating with others (Auxier and Anderson 2021; Dean 2021), and information shared in microblogs is particularly memorable to the virtual audience (Mickes et al 2013). With the growing trend towards mobile web browsing, personal status updates in microblogs have become an extremely popular form for individuals to share their everyday lived experiences online. The unique characteristics of microblogging give rise to additional unique characteristics of the registered self (see Figure 3).

The short, frequent, and real-time status updates create a presence of the person online with physical and psychological proximity, and they encourage and facilitate immediate interaction from the virtual audience through commenting, liking, reposting, and so on. This makes the registered self in microblogging constantly connected with other selves (Sheldon et al 2011). Also, status updates focus on 'what I'm doing right now', in which individuals share what they observe in their immediate surroundings and their activities, thoughts, and feelings, often using present tense (i.e., 'Enjoying coffee with Sarah'). The registered self in microblogging is, thus, temporally near the present moment. 
Furthermore, apart from being chronologically ordered and having the author as the main character, personal status updates are often seemingly isolated episodes and states of mind that the person experiences, without an apparent framework for integration. The registered self in microblogging is, therefore, sliced to reflect fragments of the person's selfhood (Page 2010; Wang 2013).

The registered self can, in turn, shape the represented self. For instance, given the performed online self-representations, it is no surprise that people feel good about themselves after viewing or editing their own online profiles while feeling bad after viewing others' posts (Faelens et al 2019; Gonzales and Hancock 2011). Frequent exposure to others' selective and glorified online self-presentations leads social media users to believe that others are happier and having better lives than they are (Chou and Edge 2012). Moreover, the registered self differentially engages the virtual audience. For instance, social media posts by those with low self-esteem tend to convey low-positivity and highnegativity content, which then elicits undesirable responses from others (Forest and Wood 2012). Social media posts with excessive presentations of the ideal self receive fewer Likes, while those with self-disclosure of personal information - a gesture that suggests eagerness to interact - receive more Likes (Hong et al 2020).

Taken together, the motives to express oneself and connect with others interact with the interface and characteristics of social media platforms to produce the registered self in cyberspace. While the person is an active agent in selectively sharing autobiographical information online, the receiver or consumer of the information, namely the virtual audience, also play an active role in inferring and working out who the person is.

\section{The inferred self and the transactive mind}

In the social media era, the represented self is not only externalised to the digital platforms to create the registered self, but also to the virtual audience to construct the inferred self (Figure 1). In the transactive mind of the virtual community, or the 'cybermind' (Wegner 1986, 2012), the audience interact with the person and among themselves and collectively encode, integrate, and update information about the characteristics, roles, and experiences of the person. The inferred self, thus, derives from information about the person shared online and operates through communicative processes within the virtual community (Wang 2013). Although the inferred self may share some characteristics with impression formation about a person in real life, it is a transactive property constructed and upheld by a large and diverse online audience and is informed by permanent and yet frequently renewed social media posts. Just like the represented self and the registered self that continue to evolve as life progresses, the inferred self continues to update as information about the person accumulates online.

Critically, the construction of the inferred self involves active meaning making by the virtual audience based on the available information about the person (Figure 4). The registered self, especially in microblogging that lists 'mere successions of doings' (Page 2010, 439), presents disparate experiences of the person, with earlier posts bearing no obvious logical connections with later ones. It is the virtual audience who interweave the slices of information into a coherent life story about who the person is. The timestamp of the online posts provides physical clues to assist the virtual audience in reconstructing a chain of events taking placing in the person's life. For family, friends, and acquaintances, their knowledge about the person in real life further helps them fill in the gaps between individual posts and interpret what is happening. Operating through the transactive memory system (Wegner 1986, 2012), the audience members further engage with each other online, exchange knowledge and information about the person, and feed their perceptions back to the community. The inferred self is constructed as 
Fig. 4. The inferred self through the transactive mind of the virtual community. Illustration by Luke Ramsey.

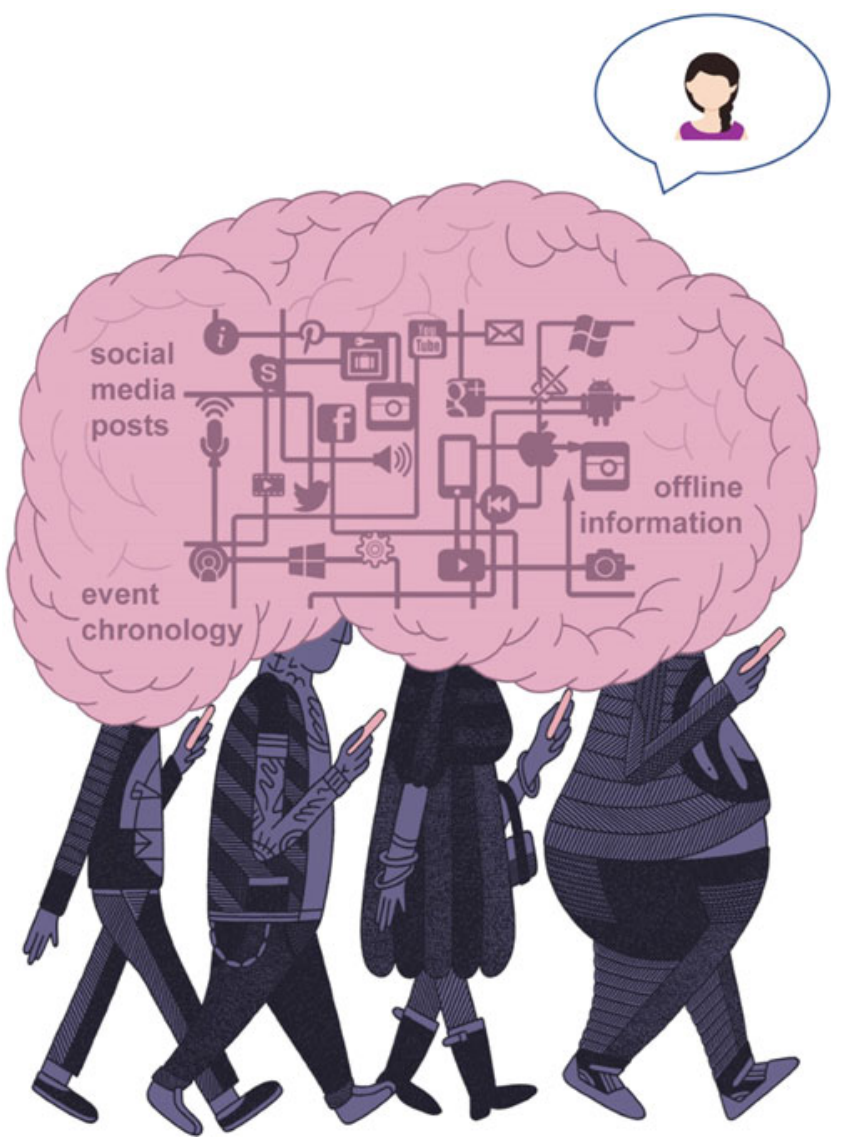

the virtual audience impose a unifying interpretive structure on the diverse posts to infer meaning and form representations of the person's selfhood (Page 2010; Wang 2013).

Not only is the inferred self constructed through the meaning-making process outsourced to the virtual audience, but it is also revised, negotiated, and updated via the audience's diverse ways of interacting with the person, such as giving Likes, commenting, and reposting. The virtual audience react to social media posts based on their knowledge of the person and interpretation of the material. The mere process of making comments on a post can shape the inferred self by boosting the commentator's memory for the post (Zimmerman and Brown-Schmidt 2020). Giving Likes to others' social media posts also appears to be rewarding for the giver, activating brain regions responsible for reward processing and prosocial behaviour (Sherman et al 2018). Interestingly, this process is reciprocal in the transactive mind of the virtual community, whereby receiving Likes on one's posts activates similar brain circuitry in the receiver as in the giver and increases the receiver's behaviour to provide positive feedback to others' posts (Davey et al 2010; Sherman et al 2016).

Based on the audience feedback or the lack of it, the person may anticipate the expectations of the audience when making future posts about themselves and further engage in self-censorship and strategic self-presentation. For instance, individuals post more frequently when they feel excited about the Likes and comments they receive, while they change the content of their future posts when they feel sad for the lack of audience response (Stsiampkouskaya et al 2021). Individuals who perceive their virtual audience 
as more responsive express themselves more openly in their social media posts (Walsh et al 2020). The frequency and content of online sharing can, in turn, shape the inferred self in the virtual audience.

By affirming or defying the person's shared experiences via their responses and presence or absence of participation, the virtual audience hold great power over not only the registered self but also the represented self. For instance, receiving a large number of Likes on one's Facebook posts boosts self-esteem, although individuals with a greater sense of purpose in life are less susceptible to social media feedback (Burrow and Rainone 2017). Receiving positive feedback on social media, especially when they come from close others, makes people feel that they are supported (Carr et al 2016). Not receiving enough 'Likes' on social media posts, on the other hand, may result in negative feelings, especially in individuals with low self-esteem and those with high levels of self-monitoring (Hou et al 2019; Scissors et al 2016). In addition, heavier social media users are more likely to believe that others share their attributes and opinions (Bunker and Varnum 2021). Pertaining to autobiographical memory, aspects of experiences that are shared with and endorsed by the virtual audience are likely to be remembered and thus remain part of the represented self (Hirst and Echterhoff 2012; Hou et al 2021).

Taken together, the construction of the inferred self is a collective process in which information about the person is perceived, negotiated, integrated, and updated through the transactive mind of the virtual community. The inferred self and the audience's reactions to the online posts further shape the sense of self of the person as well as his or her subsequent engagement with social media and the audience.

\section{Conclusion}

I have discussed a triangular theory of self in the era of social media that conceptualises the self as represented in the private mind of the person, the public sphere of social media platforms, and the transactive mind of the virtual audience. The person as an agentic knower and experiencer actively constructs the represented self - autobiographical memory in particular - and selectively externalises it online. The functional capacities and technology features of social media platforms support and constraint the registered self, facilitating the person's social and personal motives for sharing autobiographical experiences online. The virtual audience collectively construct the inferred self through meaning making of the person's diverse social media posts and through their engaging in various ways with the posts. The three components of the self mutually influence each other via dynamic, interactive, and transactive processes. Together, they constitute a sense of selfhood and identity specific to the social media era.

Acknowledgement. The author thanks the two editors for their helpful feedback.

Funding. This work received no specific grant from any funding agency, commercial, or not-for-profit sectors.

Competing interests. The author has no conflicts of interest to disclose.

\footnotetext{
Note

1 Allen M, Poggiali D, Whitaker K, Marshall TR and Kievit RA (2019) Raincloud plots: A multi-platform tool for robust data visualization [version 1; peer review: 2 approved]. Wellcome Open Research 4, 63. https://doi.org/10. 12688/wellcomeopenres.15191.1.
} 


\section{References}

Alea N and Bluck S (2003) Why are you telling me that? A conceptual model of the social function of autobiographical memory. Memory 11, 165-178.

Auxier B and Anderson M (2021) Social Media Use in 2021. Pew Research Center. Retrieved on July 29, 2021, from https://www.pewresearch.org/internet/2021/04/07/social-media-use-in-2021/.

Bell G and Gemmell J (2010) Your Life, Uploaded. New York, NY: Plume.

Bunker CJ and Varnum MEW (2021) How strong is the association between social media use and false consensus? Computers in Human Behavior 125, 106947. doi:10.1016/j.chb.2021.106947.

Burrow AL and Rainone N (2017) How many likes did I get?: Purpose moderates links between positive social media feedback and self-esteem. Journal of Experimental Social Psychology 69, 232-236. doi:10.1016/ j.jesp.2016.09.005.

Carr CT, Wohn DY and Hayes RA (2016) [Thumbs up emoji] as social support: Relational closeness, automaticity, and interpreting social support from paralinguistic digital affordances in social media. Computers in Human Behavior 62, 385-393.

Chou HTG and Edge N (2012) 'They are happier and having better lives than I am': The impact of using Facebook on perceptions of others' lives. Cyberpsychology, Behavior, and Social Networking 15, 117-121. doi:10.1089/ cyber.2011.0324.

Davey CG, Allen NB, Harrison BJ, Dwyer DB and Yucel M (2010) Being liked activates primary reward and midline self-related brain regions. Human Brain Mapping 31(4), 660-668.

Dean B (2021) Social Network Usage \& Growth Statistics: How Many People Use Social Media in 2021? Retrieved on July 2, 2021, from https://backlinko.com/social-media-users\#most-popular-social-media-platforms

Faelens L, Hoorelbeke K, Fried E, De Raedt R and Koster EHW (2019) Negative influences of Facebook use through the lens of network analysis. Computers in Human Behavior 96, 13-22. doi:10.1016/j.chb.2019.02.002.

Ferguson AM, McLean D and Risko EF (2015) Answers at your fingertips: Access to the internet influences willingness to answer questions. Consciousness and Cognition 37, 91-102.

Fivush R (2011) The development of autobiographical memory. Annual Review of Psychology 62, 559-582. doi:10.1146/annurev.psych.121208.131702.

Forest AL and Wood JV (2012) When social networking is not working: Individuals with low self-esteem recognize but do not reap the benefits of self-disclosure on Facebook. Psychological Science 23, 295-302. doi:10.1177/ 0956797611429709.

Gonzales AL and Hancock JT (2011) Mirror, mirror on my Facebook wall: Effects of exposure to Facebook on self-esteem. Cyberpsychology, Behavior, and Social Networking 14, 79-83. doi:10.1089/cyber.2009.0411.

Guan L and Wang Q (2020) Does sharing memories make us feel closer? The roles of memory type and culture. doi:10.31234/osf.io/7udy4.

Hirst W and Echterhoff G (2012) Remembering in conversations: The social sharing and reshaping of memories. Annual Review of Psychology 63, 55-79. doi:10.1146/annurev-psych-120710-100340.

Hollenbaugh EE (2011) Motives for maintaining personal journal blogs. Cyberpsychology, Behavior, and Social Networking 14(1/2), 13-20.

Hong S, Jahng MR, Lee N and Wise K (2020) Do you filter who you are?: Excessive self-presentation, social cues, and user evaluations of Instagram selfies. Computer in Human Behavior 104, 106159. doi:10.1016/ j.chb.2019.106159.

Hou Y, Xiong D, Jiang T, Song L and Wang Q (2019) Social media addiction: Its impact, mediation, and intervention. Cyberpsychology 13(1), 4. doi:10.5817/CP2019-1-4.

Hou Y, Pan X, Cao X and Wang Q (2021) Remembering online and offline: The effects of retrieval contexts, cues, and intervals on autobiographical memory. Memory, in the special issue Memory Online: Remembering in the Age of the Internet and Social. doi:10.1080/09658211.2021.1953078.

James W (1890) The Principles of Psychology. New York, NY: H. Holt and Company.

Johnson AJ and Morley EG (2021) Sharing personal memories on ephemeral social media facilitates autobiographical memory. Cyberpsychology, Behavior, and Social Networking. https://doi.org/10.1089/cyber.2020.0511.

Kahn AS and Martinez TM (2020) Text and you might miss it? Snap and you might remember? Exploring 'Google effects on memory' and cognitive self-esteem in the context of Snapchat and text messaging. Computers in Human Behavior 104, 106-166.

Kulkofsky S, Wang Q and Hou Y (2010) Why I remember that: The influence of contextual factors on beliefs about everyday memory. Memory \& Cognition 38, 461-473.

Lee D-H, Im S and Taylor CR (2008) Voluntary self-disclosure of information on the internet: A multimethod study of the motivations and consequences of disclosing information on blogs. Psychology \& Marketing 25(7), 692-710. 
Lenhart A and Fox S (2006) Bloggers: A Portrait of the Internet's New Storytellers. Pew Internet \& American Life. Retrieved on July 29, 2021, from https://www.pewtrusts.org/en/research-and-analysis/reports/2006/07/19/ bloggers-a-portrait-of-the-internets-new-storytellers.

Levinger G (1980) Toward the analysis of close relationships. Journal of Experimental Social Psychology 16(6), 510-544. doi:10.1016/0022-1031(80)90056-6.

Marr B (2021) How Much Data Do We Create Every Day? The Mind-Blowing Stats Everyone Should Read. Intelligent Business Performance. Retrieved on July 2, 2021, from https://www.bernardmarr.com/default.asp? contentID $=1438$

Mickes L, Darby RS, Hwe V, Bajic D, Warker JA, Harris CR and Christenfeld NJS (2013) Major memory for microblogs. Memory \& Cognition 41(4), 481-489. https://doi.org/10.3758/s13421-012-0281-6.

Miura A and Yamashita K (2007) Psychological and social influences on blog writing: An online survey of blog authors in Japan. Journal of Computer-Mediated Communication 12(4), 1452-1471.

Modinos G, Renken R, Ormel J and Aleman A (2011) Self-reflection and the psychosis-prone brain: An fMRI study. Neuropsychology 25(3), 295-305.

Neisser U (1988) Five kinds of self-knowledge. Philosophical Psychology 1, 35-59.

Page R (2010) Re-examining narrativity: Small stories in status updates. Text \& Talk 30(4), 423-444.

Pfeifer JH, Lieberman MD and Dapretto M (2007) "I know you are but what am I?!": Neural bases of self- and social knowledge retrieval in children and adults. Journal of Cognitive Neuroscience 19(8), 1323-1337.

Pillemer DB (2001) Momentous events and the life story. Review of General Psychology 5(2), 123-134.

Scissors L, Burke M and Wengrovitz S (2016) What's in a Like? Attitudes and behaviors around receiving likes on Facebook. In Proceedings of the 19th ACM Conference on Computer Supported Cooperative Work \& Social ComputingCSCW'16. New York: ACM Press, 1499-1508.

Serfaty V (2004) The Mirror and the Veil: An Overview of American Online Diaries and Blogs. New York: Rodopi.

Sheldon KM, Abad N and Hinsch C (2011) A two-process view of Facebook use and relatedness need-satisfaction: Disconnection drives use, and connection rewards it. Journal of Personality and Social Psychology 100(4), 766-775.

Sherman LE, Payton AA, Hernandez LM, Greenfield PM and Dapretto M (2016) The power of the like in adolescence: Effects of peer influence on neural and behavioral responses to social media. Psychological Science 27 (7), 1027-35.

Sherman LE, Hernandez LM, Greenfield PM and Dapretto M (2018) What the brain 'Likes': neural correlates of providing feedback on social media. Social Cognitive and Affective Neuroscience 13(7), 699-707. doi:10.1093/scan/ nsy051.

Sparrow B, Liu J and Wegner DM (2011) Google effects on memory: Cognitive consequences of having information at our fingertips. Science (American Association for the Advancement of Science) 333(6043), 776-778. doi:10.1126/science.1207745.

Stefanone MA and Jang C-Y (2008) Writing for friends and family: The interpersonal nature of Blogs. Journal of Computer-Mediated Communication 13(1), 123-140.

Stone CB and Wang Q (2019) From conversations to digital communication: The mnemonic consequences of consuming and producing information via social media. Topics in Cognitive Science 11(4), 774-793. doi:10.1111/ tops.12369.

Stone CB, Guan L, LaBarbera G, Ceren M, Garcia B, Huie K, Stump C and Wang Q (2021) Why do people share memories online? An examination of the motives and characteristics of social media users. Manuscript submitted to the special issue of Memory, Memory Online: Remembering in the Age of the Internet and Social Media.

Stsiampkouskaya K, Joinson A, Piwek L and Ahlbom C (2021) Emotional responses to likes and comments regulate posting frequency and content change behaviour on social media: An experimental study and mediation model. Computers in Human Behavior 124, 106940. doi:10.1016/j.chb.2021.106940.

Truong G and Todd RM (2017) SOAP opera: Self as object and agent in prioritizing attention. Journal of Cognitive Neuroscience 29, 937-952. doi:10.1162/jocn_a_01083.

Walsh RM, Forest AL and Orehek E (2020) Self-disclosure on social media: The role of perceived network responsiveness. Computer in Human Behavior 104, 106162. doi:10.1016/j.chb.2019.106162.

Wang Q (2006) Culture and the development of self-knowledge. Current Directions in Psychological Science 15(4), 182-187.

Wang Q (2013) The Autobiographical Self in Time and Culture. New York, NY: Oxford University Press.

Wang Q (2018) Culture in collaborative remembering. In Meade ML, Harris CB, Van Bergen P, Sutton J and Barnier AJ (eds), Collaborative Remembering: Theories, Research, and Applications. New York, NY: Oxford University Press, 297-314.

Wang Q (2019) The individual mind in the active construction of its digital niche. Journal of Applied Research in Memory and Cognition 8, 25-28. doi:10.1016/j.jarmac.2018.12.005. 
Wang Q (2020) Creation of the purposes of online memory sharing scale. International Journal of Applied Psychology 10(3), 65-70. doi:10.5923/j.ijap.20201003.01.

Wang Q (2021) The cultural foundation of human memory. Annual Review of Psychology 72, 151-179. doi:10.1146/ annurev-psych-070920-023638.

Wang Q and Chaudhary N (2006) The self. In Pawlik K and d'Ydewalle G (eds), Psychological Concepts: An International Historical Perspective. Hove, UK: Psychology Press, 325-358.

Wang Q, Blenis RC, Ng M and Gonzalez P (2015) Going public: The impact of social media on autobiographical memory. Poster session presented at the 27th APS Annual Convention, New York, NY.

Wang Q, Lee D and Hou Y (2017) Externalizing the autobiographical self: Sharing personal memories online facilitated memory retention. Memory 25(6), 772-776. doi:10.1080/09658211.2016.1221115.

Wegner DM (1986) Transactive memory: A contemporary analysis of the group mind. In Mullen B and Goethals GR (eds), Theories of Group Behavior. New York, NY: Springer-Verlag, 185-208.

Wegner DM (2012) Don't fear the Cybermind. New York Times. Retrieved on July 29, 2021, from https://www. nytimes.com/2012/08/05/opinion/sunday/memory-and-the-cybermind.html.

Zimmerman J and Brown-Schmidt S (2020) \#foodie: Implications of interacting with social media for memory. Cognitive Research: Principles and Implications 5(1), 16.

Qi Wang is professor of human development and psychology at Cornell University. Her research examines how cultural forces, including the Internet technology, impact autobiographical memory and the sense of self. Wang received her PhD in Psychology from Harvard University. She is the author of The Autobiographical Self in Time and Culture (OUP 2013) and Editor-in-Chief of JARMAC.cy

Cite this article: Wang Q (2022). The triangular self in the social media era. Memory, Mind \& Media 1, e4, 1-12. https://doi.org/10.1017/mem.2021.6 